\title{
PERCEPÇÕES DE FAMILIARES/CUIDADORES SOBRE INTERNAÇÃO DOMICILIAR DE PESSOAS COM DEPENDÊNCIA DE CUIDADO
}

\section{Family members'/Caregivers' perceptions about domiciliary hospitalization of people dependent on care}

\section{Percepciones de familiares/cuidadores sobre la internación domiciliaria de personas dependientes de cuidado}

\author{
Ana Paula Magalhães iD \\ Universidade do Estado da Bahia - UNEB - Salvador - (BA) - Brasil \\ Magno Conceição das Mercês (iD \\ Universidade do Estado da Bahia - UNEB - Salvador - (BA) - Brasil
}

Pablo Luiz Santos Couto iD

Centro de Ensino Superior de Guanambi - Guanambi (BA) - Brasil

Maria Lúcia Silva Servo iD

Universidade Estadual de Feira de Santana - UEFS - Feira de Santana (BA) - Brasil

Jairrose Nascimento Souza

Universidade do Estado da Bahia - UNEB - Salvador - (BA) Brasil

Marcio Costa de Souza

Universidade do Estado da Bahia - UNEB - Salvador - (BA) Brasil

Antônio Marcos Tosoli Gomes (iD

Universidade do Estado do Rio de Janeiro - UERJ - Rio de Janeiro (RJ) Brasil

Márcia Cristina Graça Marinho (iD

Universidade do Estado da Bahia - UNEB - Salvador - (BA) Brasi

\section{RESUMO}

Objetivo: Analisar as percepções de familiares/cuidadores sobre a internação domiciliar de pessoas com doenças crônicas ou em estado terminal na assistência prestada por profissionais de saúde. Métodos: Realizou-se um estudo qualitativo, entre março e abril de 2014, com entrevistas semiestruturadas, no domicílio de pacientes em internação domiciliar, com oito familiares/ cuidadores em um município da Bahia, Brasil. Analisaram-se os dados pelo método da análise de conteúdo, que permitiu a apreensão da categoria do estudo - percepções dos familiares/cuidadores de pessoas com dependência de cuidado sobre a internação domiciliar. Resultados: Os entrevistados demonstraram satisfação com a internação domiciliar, pelo cuidado diferenciado desse modo de atenção, pela redução de deslocamentos e por proporcionar conforto e interação entre equipe de saúde, familiares e a pessoa que necessita de cuidados. Além disso, o cuidado é respaldado no respeito à individualidade e à singularidade de cada família. Também viu-se que os entrevistados compreendem o quantitativo de profissionais como insuficiente, havendo necessidade de ampliar a equipe. Assim, convém sensibilizar a equipe sobre a relevância de discutir com familiares/ cuidadores sobre seus direitos como cidadãos. Conclusão: O internamento domiciliar, na percepção dos entrevistados, é uma modalidade complementar à hospitalização essencial, uma vez que a equipe domiciliar proporciona um cuidado humanizado e próximo à família. Contudo falas evidenciam que o serviço ofertado a esses pacientes parece ser um favor prestado a eles, e não um direito do paciente de receber esse serviço público de saúde.

Descritores: Percepção; Assistência Domiciliar; Família; Cuidadores.

\section{ABSTRACT}

Objective: To analyze family members'/caregivers' perceptions about domiciliary hospitalization of people with chronic or terminal illnesses receiving care from health professionals. Methods: A qualitative study was conducted between March and April 2014 using

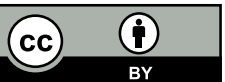


semi-structured interviews held at the households of patients in domiciliary hospitalization with eight family members/caregivers in a municipality in Bahia, Brazil. The data were analyzed using the content analysis method, which allowed the identification of the study category - family member'/caregivers' perceptions about domiciliary hospitalization of people dependent on care. Results: The interviewees reported being satisfied with domiciliary hospitalization due to the differentiated care that is provided, the reduction in hospital trips, the comfort, and the interaction between the health team, family members and the person in need of care. In addition, care is provided based on respect for the individuality and singularity of each family. The interviewees also said that the number of professionals was not enough and there was a need to expand the team. Thus, the team should be made aware of the importance of discussing the rights of family members/caregivers as citizens. Conclusion: According to the interviewees' perceptions, domiciliary hospitalization is a modality of care that is complementary to essential hospitalization as the home care team provides humanized care and is close to the family. However, the interviews showed that the service offered to these patients seems to be a favor and not a right of the patient to get this public health service.

Descriptors: Perception; Home nursing; Family; Caregivers.

\section{RESUMEN}

Objetivo: Analizar las percepciones de familiares/cuidadores sobre la internación domiciliaria de personas con enfermedades crónicas o en fase terminal para la asistencia de profesionales sanitarios. Métodos: Se ha realizado un estudio cualitativo entre marzo y abril de 2014 con entrevistas semiestructuradas en el domicilio de pacientes de internación domiciliaria con ocho familiares/ cuidadores de un municipio de Bahía, Brasil. Se analizaron los datos por el método de análisis de contenido que ha permitido la aprensión de la categoría del estudio-percepciones de los familiares/cuidadores de personas dependientes de cuidado sobre la internación domiciliaria. Resultados: Los entrevistados demostraron satisfacción con la internación domiciliaria por el cuidado de ese modo de atención, por la reducción de desplazamientos y por el conforto e interacción entre el equipo de salud, los familiares y la persona que necesita de cuidados. Además, el cuidado está respaldado en el respecto a la individualidad y la singularidad de cada familia. Se ha visto también que los entrevistados perciben el cuantitativo de profesionales como insuficiente y la necesidad de ampliar el equipo. Así, es oportuno da sensibilización del equipo sobre la relevancia de discutir con los familiares/cuidadores sobre sus derechos como ciudadanos. Conclusión: En la percepción de los entrevistados la internación domiciliaria es una modalidad complementaria de la hospitalización esencial una vez que el equipo domiciliario proporciona el cuidado humanizado y cerca de la familia. Sin embargo, algunas hablas han evidenciado que el servicio ofrecido a eses pacientes se parece a un favor y no al derecho que tienen de recibir ese tipo de servicio público de salud.

Descriptores: Percepción; Atención Domiciliaria de Salud; Familia; Cuidadores.

\section{INTRODUÇÃO}

A internação domiciliar (ID) no âmbito do Sistema Único de Saúde (SUS) é uma estratégia que compreende o conjunto de prestação de cuidados em domicílio que propicia a redução do tempo de internação hospitalar, valorizando inovações de espaços e formas de organização das tecnologias ${ }^{(1)}$. Esse serviço possibilita uma atenção humanizada, amplia o acesso ao cuidado das pessoas que necessitam de internação, além de influenciar diretamente no cotidiano dos familiares ${ }^{(2)}$.

O cuidado domiciliar remonta a milenares práticas de cuidado familiar em saúde, mas as primeiras iniciativas de regulamentação pública dessa forma de cuidado ocorreram nos Estados Unidos, na década de 40, visando à liberação de leitos hospitalares ${ }^{(3)}$. No Brasil, a primeira experiência de atenção domiciliar data do final dos anos 40 . Desde então, diferentes iniciativas foram realizadas consoante a necessidade de oferta de serviços de saúde mais compatíveis com os processos de transição demográfica e epidemiológica, com envelhecimento da população e aumento das doenças crônicodegenerativas, bem como com a perspectiva de criar alternativas para a redução dos custos com internações hospitalares ${ }^{(4)}$.

O envelhecimento da população é um fato de intensidade mundial, e as organizações internacionais estimam que, em 2025, existirão 1,2 bilhões de pessoas com mais de 60 anos, sendo que os idosos mais velhos (com 80 anos ou mais) comporão um grupo etário de significativa importância numérica ${ }^{(5)}$, contribuindo para o aumento das doenças crônicas.

As doenças crônicas são multifatoriais, de desenvolvimento insidioso e comprometem da qualidade de vida dos indivíduos, sendo as doenças cardiovasculares, os cânceres, as doenças respiratórias crônicas e a diabetes mellitus o grupo das principais doenças crônicas não transmissíveis (DCNT) $)^{(5)}$. No ano de 2012, 38 milhões de mortes ocorreram no mundo devido a DCNT, e quase $3 / 4$ destas foram registradas em países de baixa e média renda ${ }^{(6)}$. No período de 2000 a 2011, as DCNT representaram 72,7\% do total de óbitos no Brasil( ${ }^{(7)}$. 
Nesse contexto, o Ministério da Saúde instituiu a Política de Internação Domiciliar, através da Portaria n 825 , de 25 de abril de 2016, que preconiza a redução da demanda para atendimentos hospitalares ou no período de internação, na perspectiva da promoção de um cuidado mais humanizado e que fomente a ampliação da autonomia dos usuários nos seus cuidados em saúde ${ }^{(8)}$.

A partir de um conjunto de distintas estratégias, o cuidado domiciliar está entre as indicações de reorientação de práticas dos serviços de saúde, em um diálogo com a Política Nacional de Promoção da Saúde, cujo foco de atenção é transversalizar a promoção na Rede de Atenção à Saúde, promovendo práticas de cuidado humanizadas, reguladas nas necessidades locais e na integralidade do cuidado, articulando com todos os equipamentos de produção da saúde do território( ${ }^{(9)}$.

AAtenção Básica e as equipes da Estratégia Saúde da Família se responsabilizam pela execução de ações de assistência nos domicílios, entendendo o cuidado domiciliar como um seguimento da atenção integral em saúde e mais próximo das necessidades dos usuários ${ }^{(10)}$.

A internação domiciliar emerge como modalidade de cuidado com a finalidade de suprir as necessidades atuais de cuidado permanente de pessoas com doenças crônicas ou em estado terminal, evitando as internações hospitalares, que as expõem a maior susceptibilidade de adquirir infecções e as distanciam do ambiente familiar ${ }^{(11)}$.

O cuidado domiciliar desponta, assim, como alternativa importante para a promoção da saúde e qualidade de vida de quem apresenta limitações ou dependência de cuidado. Nesse âmbito, o cuidador contribui sobremaneira na assistência das necessidades individuais da pessoa dependente, tornando-se, desse modo, sujeito e ator das ações em saúde ${ }^{(12)}$. Cuidador é a pessoa, com ou sem vínculo familiar com o usuário, habilitada para auxiliá-lo em suas necessidades e atividades da vida cotidiana ${ }^{(13)}$.

Tais considerações justificam a importância de se estudar a temática em questão. Portanto, emerge como pressuposto deste estudo: Como será que o familiar/cuidador percebe a assistência à internação domiciliar prestada por profissionais de saúde a seu parente/ pessoa que ele cuida com doenças crônicas ou em estado terminal? Assim, o estudo será relevante, pois, através dessas percepções, a equipe de saúde poderá repensar sua prática, considerando a possibilidade de haver maior aproximação de seu meio social, o domicílio, bem como contribuir para uma melhor compreensão do processo de pacientes graves, como em coma, tanto para o familiar/cuidador quanto para a equipe de saúde.

Assim, o presente estudo tem como objetivo analisar as percepções de familiares/cuidadores sobre a internação domiciliar de pessoas com doenças crônicas ou em estado terminal na assistência prestada por profissionais de saúde.

\section{MÉTODOS}

Trata-se de uma pesquisa de abordagem qualitativa, fundamentada na compreensão mais aprofundada do universo dos distintos significados dos fenômenos no mundo social. A abordagem qualitativa fomenta o desvelamento das experiências, das narrativas e dos fatos sociais sob a luz dos processos de subjetivação dos pacientes implicados ${ }^{(14)}$. A adoção dessa abordagem se justifica por sua compatibilidade com os objetivos propostos, isto é, de capturar as dimensões imateriais dos significados e percepções dos participantes da pesquisa acerca dos cuidados na internação domiciliar, a partir das experiências concretas vivenciadas por estes no cuidado de seus familiares/pacientes.

Realizou-se o estudo na cidade de Guanambi, Bahia, Brasil, no período de março a abril de 2014. O município de Guanambi é situado no sudoeste da Bahia, que dista $796 \mathrm{~km}$ da capital. O município possui 78.801 habitantes ${ }^{(15)}$, tem clima semiárido e constitui-se em polo regional de economia, saúde e educação ${ }^{(16)}$. O cenário desta pesquisa foi o Hospital Regional de Guanambi (HRG). Trata-se de um hospital geral, público, conveniado com o campus XII da Universidade do Estado da Bahia (Uneb) para realização dos estágios curriculares do curso de Enfermagem, sendo centro de referência microrregional em saúde com 140 leitos. É referência de atendimento à gestação de alto risco, às urgências e emergências para 31 municípios. Possui ambulatório especializado e de segmento, além de contar com recursos humanos e tecnológicos para a prestação de assistência médica de média complexidade nas clínicas médica, cirúrgica, pediátrica e obstétrica, e internamento em UTI adulto, UTI Neonatal e Neurocirurgia(17).

Escolheu-se este cenário devido ao vínculo da equipe multiprofissional do programa de internação domiciliar, vinculado ao Hospital Regional de Guanambi, que desenvolve esse serviço desde o ano de 2012, buscando adotar mecanismos para qualificar a assistência prestada aos usuários do Sistema Único de Saúde (SUS) e reduzir a sobrecarga nos grandes hospitais púbicos ${ }^{(17)}$. O programa, por ocasião da pesquisa, tinha 60 pacientes cadastrados e todos apresentavam mobilidade reduzida e a maioria tinha sequelas de doenças crônicas não transmissíveis (DCNT). 
A coleta de dados deu-se por entrevista semiestruturada, considerada como a técnica ideal utilizada para a coleta de dados neste estudo qualitativo, porque permite, a partir das experiências particulares dos participantes implicados, a evocação dos modos de pensar e agir ${ }^{(18)}$. As entrevistas foram realizadas em local reservado, nos domicílios, com os familiares/cuidadores, o que constitui o local das entrevistas. Estas foram gravadas e, posteriormente, transcritas na íntegra. Tiveram a duração de 30 minutos cada, após permissão dos participantes do estudo, preservando a fidedignidade dos relatos. Houve interação entre entrevistado e entrevistador.

Fizeram parte do estudo os familiares/cuidadores de pessoas com doenças crônicas ou em estado terminal, após receberem o convite verbal para participar do estudo e informações sobre os objetivos da pesquisa, que preencheram os seguintes critérios: idade igual ou superior a 18 anos, cuidador principal no domicílio, cônjuge ou pessoa com parentesco de primeiro ou segundo grau, residente na cidade em que o estudo foi desenvolvido e cuidador de pessoas com doenças crônicas ou em estado terminal com tempo de internação superior ou igual há 30 dias. Não se incluíram os participantes que não atenderam aos critérios de participação mencionados e os que não desejaram fazer parte do estudo. Ressalta-se, entretanto, que não houve nenhuma pessoa que se negasse a participar do estudo. Assim, a amostra total compunha-se por oito familiares/cuidadores. Destaca-se que o encerramento da amostra ocorreu por saturação de dados ${ }^{(19)}$.

As entrevistas continham um bloco de perguntas relacionadas à identificação de cada entrevistado (familiar/ cuidador) nas questões iniciais, com as variáveis sexo, idade, estado civil, escolaridade, ocupação e renda familiar. Em seguida, a questão norteadora, relacionada ao objetivo do estudo: Como você percebe a assistência a internação domiciliar prestada por profissionais de saúde a seu parente/ pessoa que você cuida?

Para o tratamento dos dados, utilizou-se o método da análise de conteúdo ${ }^{(20)}$. Dividiu-se o plano de análise em três fases: pré-análise, exploração do material, tratamento dos resultados e interpretação. Após leitura criteriosa das entrevistas de forma horizontal e vertical, apreenderam-se os núcleos de significação (satisfação, cuidado, apoio, agradecimento, empenho, domicílio, equipe de saúde, dificuldade de deslocamento), os quais permitiram a apreensão da categoria do estudo: Percepções dos familiares/cuidadores de pessoas com dependência de cuidado sobre a internação domiciliar (ID).

Este estudo foi encaminhado ao Comitê de Ética e Pesquisa da Universidade do Estado da Bahia - CEP/UNEB aprovado sob o Parecer $n^{\circ}$ 558.697. Os participantes assinaram o Termo de Consentimento Livre e Esclarecido (TCLE). Para a identificação e diferenciação das distintas falas, utilizaram-se nomes de pedras preciosas para os familiares/cuidadores, preservando a identidade dos participantes do estudo.

\section{RESULTADOS E DISCUSSÃO}

Neste espaço será apresentada a identificação dos familiares/cuidadores e, em seguida, discutida a categoria Percepções dos familiares/cuidadores de pessoas com dependência de cuidado sobre a internação domiciliar (ID) como espaço de relações, interações e qualidade de vida.

\section{Identificação dos familiares/cuidadores}

Em relação aos familiares/cuidadores, caracterizam-se por ser a maioria pertencente ao sexo feminino. No que se refere à idade, seis possuem idade maior que 50 anos e dois têm idade inferior a 50 anos. Quanto ao estado civil, cinco entrevistados eram casados, um referiu ser solteiro e duas são viúvas. No que se refere à escolaridade, nota-se que um referiu não ter alfabetização, quatro têm o $1^{\circ}$ grau incompleto, um possui $2^{\circ}$ grau incompleto e dois apresentam $2^{\circ}$ grau completo. Na caracterização ocupacional, cinco indivíduos entrevistados são aposentados, um é pensionista, um é autônomo e um é estudante. Sobre a renda mensal familiar, oito recebem até três salários mínimos.

\section{Percepções dos familiares/cuidadores de pessoas com dependência de cuidado sobre a internação domiciliar (ID)}

A categoria Percepções dos familiares/cuidadores de pessoas com dependência de cuidado sobre a internação domiciliar (ID) apresenta o familiar/cuidador no contexto da ID, sendo considerado um elo interativo entre a pessoa com doença crônica, ou em estado terminal, e a equipe de saúde.

É importante conhecer suas vivências e experiências na ID, a fim de que, a partir desses conhecimentos, os profissionais de saúde possam contribuir, efetivamente, para uma prática inovadora de educação e de cuidado à saúde, inserindo os envolvidos nesse processo( ${ }^{(21)}$.

O relato a seguir evidencia que a percepção dos entrevistados sobre o ID tem a ver com "merecimento", "dádiva de Deus": 
"[...] sinto-me muito satisfeita, sinto muito orgulho de ter esse merecimento, de receber essas meninas aqui, na minha casa, todo dia... Para falar a verdade, nunca pensei... Mas Deus viu que eu estava precisando!" (TURQUESA)

Essa fala evidencia que é possível inferir e que essa entrevistada parece não possuir um conhecimento dos seus direitos como cidadã, uma vez que não reconhece ser dever do governo garantir o direito à ID. Pelo princípio de universalidade do SUS, o indivíduo passa a ter direito ao acesso a todos os serviços públicos de saúde ${ }^{(22)}$. A dádiva divina pode ser caracterizada, para ela, como privilégio, com a consequente associação de receber cuidados da equipe profissional, e não à estrutura e à organização do sistema em si. Essas outras falas, seguem elogiando o serviço recebido:

"[...] acho que todas que vêm, cuidam bem, veem que ela está em casa, não tem nada melhor que isso para um filho [...]. No hospital, é muito mais difícil [...] Esse negócio de internação em casa foi a melhor coisa." (CRISTAL)

"[...] nós estamos satisfeitos, eu e a minha esposa. A gente, assim... até agradece por esse trabalho, todo esse empenho de o governo estar liberando essa internação domiciliar. É um trabalho muito bom." (DIAMANTE)

Os pacientes atendidos no programa de ID eram pessoas idosas, que passaram grande parte de suas vidas sem ter esse cuidado integral e estruturado no âmbito das Redes de Atenção à Saúde, conforme preconiza a Portaria n 963/2013, que redefine a atenção domiciliar no Sistema Único de Saúde ${ }^{(13)}$. Dessa forma, não estão acostumados com esse tipo de atendimento. As falas se direcionam, também, para uma percepção positiva, demonstrada pela satisfação com o cuidado no domicílio, uma vez que, para eles, isso significa conforto, tranquilidade, garantia de um atendimento humanizado e personalizado, voltado para as reais necessidades das pessoas que requerem cuidados permanentes.

Em um estudo realizado em 2014, com pacientes em ID, a avaliação de familiares/cuidadores condiz com os achados no presente estudo. Os entrevistados responderam que o cuidado em casa significa proteção, com melhoria na qualidade de vida, a partir de uma atenção humanizada por parte dos profissionais ${ }^{(23)}$.

Como estratégia inovadora, a implementação da ID requer reflexão no que se refere à organização das práticas, considerando os envolvidos, a integralidade do cuidado, os recursos necessários para desenvolver esse cuidado, assim como a articulação com os demais serviços de saúde. Desse modo, esse tipo de atenção deve ser visto como uma importante estratégia, e não somente uma alternativa oferecida a pessoas que são transferidas o mais rápido possível para seus domicílios, com intuito de recuperação, reabilitação ou de cuidados paliativos, mas que possa ser, de fato, visto com um espaço de cuidado, de forma integral e humanizada( ${ }^{(24)}$.

Vale ressaltar que os cuidados paliativos são definidos pela Organização Mundial da Saúde (OMS) como ações oferecidas a pessoas portadoras de doenças que não respondem mais ao tratamento curativo. Dessa forma, o controle da dor e outros sintomas, a atenção relacionada a questões psicológicas e o suporte emocional e social são os mais relevantes, os quais têm como principal objetivo melhorar a qualidade de vida dos pacientes e sua família ${ }^{(25)}$.

Em seus relatos, os participantes percebem a importância do cuidado multiprofissional no domicilio, como receber a equipe que Ihes dá conforto, apoio emocional, reabilitação, cuidados de enfermagem e orientação nutricional. É o que retratam as falas a seguir:

"[...] ela sente falta. Hoje mesmo, ela estava perguntando: cadê a enfermeira que não veio aqui? Eu falei: 'oh, mãe, amanhã. Elas vêm duas vezes na semana'. Até ela se sente bem com o trabalho de vocês." (CRISTAL)

"Eu gostei. Elas, as profissionais, são muito boas, educadas, cumprem o horário de injeção... Não tenho o que falar mesmo [...] Fiquei muito satisfeita... Está sendo ótimo, estar sendo atendido em casa é outra coisa." (SAFIRA)

"[...] elas são profissionais boas. Chegam, fazem um curativo e conversam com ele. Todas vêm e fazem o trabalho delas, ele gosta bastante. Passam as receitas, pedem exames, vem a médica, a outra vem [...] conversa com ele." (RUBI)

"[...] tinha coisa que ela não podia comer. [...] Aí, veio a nutricionista, [...] veio a fisioterapeuta também... Todas as orientações eu tenho. Às vezes, tem hora que eu esqueço alguma coisa, aí elas chegam na semana, eu pergunto a elas, e me ensinam, ali, o que é que eu vou fazer. Então, graças a Deus, foi muito bom para mim." (ESMERALDA)

Viu-se, nas falas dos entrevistados, que ocorre o direcionamento de um cuidado compartilhado entre familiar/ cuidador e trabalhadores de saúde, que se configura numa possibilidade do estabelecimento de relações horizontais, 
com a valorização do outro que não faz parte da equipe de saúde, mas que é participante ativo no processo de cuidar na ID. São aspectos relevantes que devem ser ressaltados na ID e que são diferentes da realidade da prática hospitalar, centrada, principalmente, na figura do médico. Sabe-se que está longe da construção de projetos terapêuticos singulares, mas há avanços. Os familiares/cuidadores entrevistados expõem que a experiência com a assistência domiciliar é muito positiva. Eles percebem que essa modalidade de atendimento faz diferença, reconhecendo a importância das visitas da equipe de saúde, tanto para a família quanto para a pessoa com doença crônica ou em estado terminal.

A equipe de saúde, ao exercer o cuidado no domicílio, o faz de uma maneira diferenciada, pois há um envolvimento diferente daquele estabelecido no hospital, e a família gosta de ser cuidada em casa, não apenas pelo conforto proporcionado, mas, também, pelo cuidado diferenciado que recebe ${ }^{(26)}$.

Dessa forma, à medida que a família recebe orientação profissional para execução dos cuidados diários ao paciente, sente-se mais tranquila e tende a diminuir o seu nível de estresse ${ }^{(27)}$.

Os familiares/cuidadores verbalizaram, ainda, que o Programa de ID ao possibilitar receber a equipe de saúde em casa, se constitui em vantagem significativa devido a facilidades de atendimento e a não necessidade de deslocamento da pessoa com doença crônica ou em estado terminal, evitando o desgaste e possibilitando a otimização do tempo e dos recursos. Os fragmentos das falas abaixo traduzem tais afirmativas:

"A internação domiciliar foi um projeto muito bom que o governo implantou, porque não temos condições de levar, estar sempre se deslocando com ele. A gente está sem carro. [...] Imagina levar uma criança, de manhã, ao fisioterapeuta; de tarde, ao fonoaudiólogo; depois, para tirar sangue... Então, quer dizer... iria ser um desgaste bem maior para levar a criança lá e cá, então eu acho que isso aí ajuda demais!" (DIAMANTE)

"[...] É uma vantagem muito boa, porque... Não precisa... Agora mesmo, nesse estado, para mim, estar me deslocando com ela para a Regional, eu não tenho condição, não. [...] Aí, eu tenho o internamento domiciliar, que vem em casa. [...] Se você precisar, pode ligar. Eu já liguei várias vezes para lá e eles atendem." (ESMERALDA)

Nesse sentido, o serviço de ID pode se caracterizar como um suporte indireto às limitações sociais e econômicas dos familiares/cuidadores, colaborando para a eliminação de gastos e a economia do tempo que estaria envolvido no processo de deslocamento da pessoa com doença crônica ou em estado terminal. Outros aspectos a se considerar como de potencial favorável dessa modalidade de prestação de cuidado se refere à redução da longa espera em um serviço de pronto atendimento, isto é, à facilidade de acesso à equipe de saúde, sendo ela referência para essa pessoa que requer cuidado permanente, o que acarreta, de certa forma, facilidade no atendimento à emergência e urgência ${ }^{(28)}$.

No que se refere aos recursos humanos do programa de ID do município estudado, são sinalizadas, nas falas dos familiares/cuidadores de pessoa com doença crônica ou em estado terminal, a falta de alguns profissionais ou de determinados serviços que auxiliariam ou facilitariam a acessibilidade do paciente a serviços complementares. É o que se apreende nas falas a seguir:

“[...] eu acredito que se melhorasse a quantidade de profissionais, até para que os outros profissionais, que já estão no trabalho, pudessem ter mais tempo para cada paciente, seria melhor. [...] A gente está sentindo falta de alguns outros profissionais, [...] que é um psicólogo, e outras áreas, não é? Aí seria bom demais." (DIAMANTE)

"Do jeito que está, acho que está bom. Mas acho que precisava era aumentar a quantidade de médicos." (ESMERALDA)

"Ter mais profissionais, [por]que não tem tantos... São poucos profissionais para muito pacientes. Eu acho muito pouco. [...]" (AMETISTA)

Apesar da satisfação com o tipo de atenção prestada à comunidade, foi possível observar que os familiares/ cuidadores sentem a necessidade da ampliação do número de profissionais que cuidam, bem como a necessidade de inserção de outras categorias profissionais. Esses achados condizem com os de uma pesquisa realizada com 14 familiares/cuidadores de um programa de atenção domiciliar vinculado ao hospital público em um município baiano, através de uma entrevista semiestruturada, que apontou também a falta de profissionais, a exemplo do psicólogo ${ }^{(24)}$.

Corroborando as falas anteriormente apresentadas, tanto as pessoas que requerem cuidados permanentes como os familiares/cuidadores envolvidos na ID demonstram interesse na ampliação da equipe de assistência domiciliar, necessitando de mais profissionais engajados nesse tipo de atendimento. Um acréscimo que tanto se dirige à perspectiva de quantidade de profissionais quanto ao acesso a outros campos disciplinares, com saberes e práticas que também constroem os cuidados em saúde, em distintas dimensões, e que vão além da atenção 
biomédica, desdobrando-se nos cuidados psicossociais, o que permite favorecer a integralidade no cuidado em saúde desse perfil de público(29).

O atual estudo confirma a pertinência da Política Nacional de Promoção da Saúde ${ }^{(9)} e$ as diretrizes da Atenção Domiciliar ${ }^{(8)}$ por demonstrar que o acesso ao cuidado, em seus diferentes níveis, na Rede de Atenção, especialmente para os pacientes em situação de maior necessidade de assistência, pode representar a vivência de uma condição de saúde-doença complexa com dignidade e respeito à singularidade das condições físicas dos pacientes e às vicissitudes sociais das famílias.

As limitações do presente estudo estão relacionadas ao fato de ter sido desenvolvido em um único serviço de saúde, com singularidades próprias. Contudo oportuniza evidenciar que pode servir de subsídio ao delineamento de políticas públicas, não apenas no município objeto deste estudo, mas em outros locais do país.

O impacto do atual estudo é que ele colabora na construção de evidências sobre a importância da internação domiciliar e demonstra que a qualidade da prestação do cuidado estruturado pelos profissionais de saúde se pauta nas necessidades dos usuários, considerando, em particular, a especificidade do perfil de morbidade dos pacientes, proporcionando conforto e qualidade de vida. A internação domiciliar, a partir dessa experiência concreta, mostra-se capaz de constituir uma opção assistencial com foco na humanização da assistência e na conexão com pontos de atenção na Rede de Atenção à Saúde (no caso, na rede hospitalar), o que permite fortalecer as diretrizes das políticas de Atenção Domiciliar, que, entre outros aspectos, busca promover a emergência de outros enfoques não tradicionais na atenção à saúde ${ }^{(30)}$.

\section{CONSIDERAÇÕES FINAIS}

As percepções reveladas por familiares/cuidadores de pessoas com doença crônica ou em estado terminal sobre a ID sinalizam: a satisfação com o atendimento ofertado pela equipe domiciliar; a melhoria da relação e interação entre o familiar/cuidador, a pessoa que requer cuidado e o profissional da equipe de saúde com o cuidado diferenciado que se encontra pautado no respeito à individualidade e à singularidade de cada família; bem como a não necessidade de deslocamento dessa pessoa. Contudo, percebe-se, também, pelas falas, que o serviço ofertado a esses pacientes parece ser um favor prestado a eles, e não um direito de acesso ao serviço público de saúde. Além disso, viu-se que os entrevistados acham o quantitativo de profissionais insuficientes, havendo necessidade de ampliar a equipe. Assim, convém sensibilizar a equipe sobre a relevância de discutir com familiares/cuidadores sobre seus direitos como cidadãos.

A prática de cuidado envolve relações entre pessoas em processo de aprendizado e de subjetivação mútuos, o que implica, ainda, considerar para novos estudos o lugar ocupado pelos familiares/cuidadores nessa tríade, o quanto interferem no cuidado prestado e como a entrada de profissionais de saúde na cena familiar pode representar mudanças de papéis familiares e novos arranjos de cuidado.

\section{CONFLITOS DE INTERESSE}

Autores declaram que não houve conflitos de interesses na concepção deste trabalho.

\section{CONTRIBUIÇÕES}

Todos os autores contribuíram com a concepção do trabalho; a coleta, organização, análise e interpretação dos dados; a redação do artigo e a versão final a ser publicada.

\section{REFERÊNCIAS}

1. Yavo IS, Campos EMP. Cuidador e cuidado: o sujeito e suas relações no contexto da assistência domiciliar. Rev Psico: Teoria e Cuidado [Internet]. 2016 [acesso em 2019 Jan 11];17(3):20-32. Disponível em: http:// pepsic.bvsalud.org/pdf/ptp/v18n1/02.pdf

2. Oliveira SG, Quintana AM, Dernardin-Budó ML, Luce-Kruse MH, García RP, Wünsh S, et al. Representações sociais do cuidado de doentes terminais no domicílio: o olhar do cuidador familiar. Aquichan [Internet]. 2016 [acesso em 2019 Jan 11];16(3):359-69. Disponível em: http://www.scielo.org.co/pdf/aqui/v16n3/v16n3a07.pdf

3. Braga PP, Sena RR, Seixas CT, Castro EAB, Andrade AM, Silva YC. Oferta e demanda na atenção domiciliar 
em saúde. Ciênc Saúde Coletiva [Internet]. 2016 [acesso em 2019 Abr 27];21(3):903-12. Disponível em http://www.scielo.br/scielo.php?script=sci_arttext\&pid=S1413-81232016000300903\&lng=en.http://dx.doi. org/10.1590/1413-81232015213.11382015

4. Ministério da Saúde (BR). Secretária de Atenção à Saúde. Departamento de Atenção Básica. Caderno de atenção domiciliar. Brasília: Ministério da Saúde; 2012.

5. Goulart FAA. Doenças crônicas não transmissíveis: estratégias de controle e desafios e para os sistemas de saúde [Internet]. Brasília: Organização Pan-Americana de Saúde; 2011. [acesso em 2019 Abr 29]. Disponível em: https://www.nescon.medicina.ufmg.br/biblioteca/imagem/4857.pdf

6. World Health Organization. Global Status Report on noncommunicable diseases 2014. Switzerland: World Health Organization; 2014.

7. Malta DC, Moura L, Prado RR, Escalante JC, Schmidt MI, Duncan BB. Mortalidade por doenças crônicas não transmissíveis no Brasil e suas regiões, 2000 a 2011. Epidemiol Serv Saúde [Internet]. 2014 [acesso em 2019 Abr 26];23(4):599-608. Disponível em: http://www.scielo.br/scielo.php?script=sci_arttext\&pid=S2237$96222014000400599 \& \operatorname{lng}=e n$

8. Brasil. Ministério da Saúde. Portaria $n^{\circ} 825$, de 25 de abril de 2016. Redefine a Atenção Domiciliar no âmbito do Sistema Único de Saúde (SUS) e atualiza as equipes habilitadas [Internet]. Diário Oficial da União; Brasília, 26 Abril de 2016. [acesso em 2019 Abr 29]. Disponível em: http://www.in.gov.br/web/guest/materia/-/ asset_publisher/Kujrw0TZC2Mb/content/id/22685962/do1-2016-04-26-portaria-n-825-de-25-de-abrilde-2016-226

9. Brasil. Ministério da Saúde. Secretaria de Vigilância em Saúde, Secretaria de Atenção à Saúde. Política Nacional de Promoção da Saúde: PNPS: revisão da Portaria MS/GM nº 687, de 30 de março de 2006. Brasília: Ministério da Saúde; 2015.

10. Savassi LCM. Os atuais desafios da Atenção Domiciliar na Atenção Primária à Saúde: uma análise na perspectiva do Sistema Único de Saúde. Rev Bras Med Fam Comunidade [Internet]. 2016 [acesso em 2019 Abr 09];11(38):1-12. Disponível em: http://dx.doi.org/10.5712/rbmfc11(38)1259

11. Muniz EA, Freitas CASL, Oliveira EN, Lacerda MR. Grau de sobrecarga dos cuidadores de idosos atendidos em domicílio pela Estratégia Saúde da Família. Saúde Debate [Internet]. 2016 [acesso em 2019 Jan 11];40(110):172-82. Disponível em: http://www.scielo.br/pdf/sdeb/v40n110/0103-1104-sdeb-40-110-0172.pdf

12. Carvalho DP, Toso BRGO, Viera CS, Garanhani ML, Rodrigues RM, Ribeiro LFC. Ser cuidador e as implicações do cuidado na atenção domiciliar. Texto Contexto Enferm [Internet]. 2015 [acesso em 2019 Jan 11];24(2):450-8. Disponível em: http://www.scielo.br/pdf/tce/v24n2/pt_0104-0707-tce-24-02-00450.pdf

13. Brasil. Ministério da Saúde. Portaria $n^{\circ} 963$, de 27 de maio de 2013. Redefine a Atenção Domiciliar no âmbito do Sistema Único de Saúde (SUS) [Internet]. 2016 [acesso em 2019 Abr 29]. Disponível em: http://bvsms. saude.gov.br/bvs/saudelegis/gm/2013/prt0963_27_05_2013.html

14. Minayo MCS. Ciência, técnica e arte: o desafio da pesquisa social. In: Deslandes SF, Cruz O Neto, Gomes R, Minayo MCS, organizadores. Pesquisa social. Teoria método e criatividade. Petrópolis: Vozes; 1994.

15. Instituto Brasileiro de Geografia e Estatística. Censo Demográfico 2010 [Internet]. 2011 [acesso em 2019 Abr 30]. Disponível em: http://www.censo2010.ibge.gov.br

16. Prefeitura de Guanambi. A cidade [Internet]. 2017 [acesso em 2019 Abr 30]. Disponível em: http://www. guanambi.ba.gov.br/texto/a_cidade

17. Governo do Estado (BA). Secretaria de Saúde. Hospital Regional de Guanambi [Internet]. 2018 [acesso em 2019 Abr 30]. Disponível em: http://www.saude.ba.gov.br/hospital/hospital-regional-de-guanambi/

18. Fontanella BBJ, Magdaleno R Júnior. Saturação teórica em pesquisas qualitativas: contribuições psicanalíticas. Psicologia em Estudo. 2012;17(1):63-71.

19. Deslauries JP. Recherche Qualitative Guide Pratique. Thema: Canadá; 1991.

20. Bardin L. Análise de Conteúdo. Lisboa: Edições 70; 2011.

21. Pereira LSM, Soares SM. Fatores que influenciam a qualidade de vida do cuidador familiar do idoso com 
demência. Ciênc Saúde Colet [Internet]. 2015 [acesso em 2019 Jan 11];20(12):3839-51. Disponível em: http:// www.scielo.br/pdf/csc/v20n12/1413-8123-csc-20-12-3839.pdf

22. Cunha JPP, Cunha RRE. Sistema Único de Saúde - SUS: princípios. In: Campos FE, Oliveira M Jr, Tonon LM, organizadores. Cadernos de saúde I. Planejamento e gestão em saúde. Belo Horizonte (MG): Coopmed; 1998. p. 11-26.

23. Fogaça NJ, Carvalho MM, Montefusco SRA. Percepções e sentimentos do familiar/cuidador expressos diante do ente em internação domiciliar. Rev Rene [Internet]. 2015 [acesso em 2019 Fev 18];16(6):848-55. Disponivel em: https://www.redalyc.org/articulo.oa?id=324043261012

24. Espíndola MMM, Carvalho MFAA, Carvalho e Lira MOS, Moura LTR, Silva RM. Assistência domiciliar à saúde - percepção do familiar cuidador sobre a qualidade assistencial. Rev Enferm UFPE on line [Internet]. 2014 [acesso em 2019 Jan 11];8(2):379-84. Disponível em: https://periodicos.ufpe.br/revistas/revistaenfermagem/ article/viewFile/9685/9734

25. Simão VM, Mioto RCT. O cuidado paliativo e domiciliar em países da América Latina. Saúde Debate [Internet]. 2016 [acesso em 2019 Jan 11];40(108):156-69. Disponível em: http://www.scielo.br/pdf/sdeb/ v40n108/0103-1104-sdeb-40-108-00156.pdf

26. Juan-Pocar M, Guillamón-Gimeno L, Pedraz-Marcos A, Palmar-Santos AM. Cuidado familiar de pessoas com doenças mentais graves: uma revisão integrativa. Rev Latino-Am Enfermagem [Internet]. 2015 [acesso em 2019 Jan 11];23(2):352-60. Disponível em: http://www.scielo.br/pdf/rlae/v23n2/pt_0104-1169rlae-23-02-00352.pdf

27. Neves ACOJ, Castro EAB, Costa SRD. Necessidades de cuidados domiciliares de enfermagem após a alta hospitalar no contexto do SUS. Cogitare Enferm [Internet]. 2016 [acesso em 2019 Jan 11];21(4):1-10. Disponível em: http://docs.bvsalud.org/biblioref/2016/12/827309/47708-191967-1-pb.pdf

28. Piolli KC, Medeiros M, Sales CA. Significações de ser cuidadora do companheiro com câncer: um olhar existencial. Rev Bras Enferm [Internet]. 2016 [acesso em 2019 Jan 11];69(1):99-105. Disponível em: http:// www.scielo.br/pdf/reben/v69n1/0034-7167-reben-69-01-0110.pdf

29. Loureiro LSN, Pereira MA, Fernandes MGM, Oliveira JS. Percepção de enfermeira sobre a tensão do papel do cuidador. Rev Baiana Enferm [Internet]. 2015 [acesso em 2019 Jan 11];29(2):164-71. Disponível em: https://portalseer.ufba.br/index.php/enfermagem/article/view/12596/pdf_122

30. Brasil. Ministério da Saúde. Atenção Domiciliar no SUS: resultados do laboratório de inovação em atenção domiciliar. Brasília: Ministério da Saúde; 2014.

\author{
Endereço do primeiro autor: \\ Ana Paula Magalhães \\ Universidade do Estado da Bahia - UNEB \\ Departamento de Ciências da Vida \\ Rua Silveira Martins, 2555 \\ Bairro: Cabula \\ CEP: 41150-000 - Salvador - BA - Brasil \\ E-mail: paulinha_tn09@hotmail.com \\ Endereço para correspondência: \\ Magno Conceição das Mercês \\ Universidade do Estado da Bahia - UNEB \\ Departamento de Ciências da Vida \\ Rua Silveira Martins, 2555 \\ Bairro: Cabula \\ CEP: 41150-000 - Salvador - BA - Brasil \\ E-mail: mmerces@uneb.br
}

Como citar: Magalhães AP, Mercês MC, Couto PLS, Servo MLS, Souza JN, Souza MC, et al. Percepções de familiares/ cuidadores sobre internação domiciliar de pessoas com dependência de cuidado. Rev Bras Promoç Saúde. 2019;32:8881. 